Pure Sciences

Poster

Abstract ID: 100

\title{
The mechanistic role of catalytic residues in non-stereo dehalogenase (DehE)
}

Azzmer Azzar Abdul Hamid|Muhammad Hasanussin

Kulliyyah of Science, International Islamic University Malaysia

Introduction: DehE or non-stereospecific dehalogenase from Rhizobium sp. RC1 catalyzes the hydrolytic dehalogenation of both isomers of $\mathrm{D}, \mathrm{L}$-2-haloacids to produce an inverted configuration of 2-hydroxyl acid. The enzymatic degradation for the removal of halides from haloacid substance has been highlighted since the enzyme itself is a potential candidate as bio-remediation agent. However, the mechanistic role of active site residues for dehalogenase is still unclear, especially for non-stereospecific ones. Method: In this study, using computational analysis, the complex structure of DehE docked with catalytic water (DehE- $\mathrm{H}_{2} \mathrm{O}$ ) was subjected to MD simulation to study the mechanistic role of catalytic residues, especially Asn114 and Asp189 towards catalytic water. Results: Our findings confirmed that Asn114 hold the catalytic water at distance of $\sim 4 \AA$ while Asp189 was appropriately oriented towards catalytic water for catalysis reaction throughout the simulation process. Conclusions: The results attained here will play important role in elucidating the direct attack mechanism of halogenated compound by non-stereospecific haloacid dehalogenase. Moreover, this research could suggest different solvent that can be used in dehalogenation to produce a beneficial product other than hydroxyl acid.

KEYWORDS: DehE, MD simulation, haloacid dehalogenase, catalytic mechanism 